\title{
Development of Epoxy-polyester Nanocomposite Materials with Improved Physical and Mechanical Properties for Increasing Transport Vehicle Reliability
}

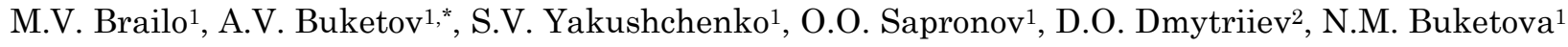 \\ ${ }^{1}$ Kherson State Maritime Academy, 20, Ushakov Ave., 73003 Kherson, Ukraine \\ ${ }^{2}$ Kherson National Technical University, 24, Beryslavske Shose, 73008 Kherson, Ukraine
}

(Received 30 October 2020; revised manuscript received 15 February 2021; published online 25 February 2021)

\begin{abstract}
The significant attention is paid to the development of new composite materials which can be widely used in the manufacturing and repairing details and their components in different branches of industry including water transport. Intensity of cargo transportation by the water transport, an increase in the frequency of loading operations, their operation under the influence of aggressive environments put forward requirements for the improvement of indicators of resource saving of vehicle units and increasing the interresource period of work. In this context, the epoxy composite materials are one of the most promising materials, which meet the requirements of modern transport industry, due to their performance characteristics, which in most cases are better than traditional metals and alloys. Therefore, in the work, the physical and mechanical properties of composite materials with nanodispersed additives based on the epoxypolyester matrix, were studied. An oxidized nanoparticle additive and pyrogenic silicon dioxide were used as fillers. It was found that epoxy-polyester composites are characterized by improved properties at significant amount of the oxidized nanoparticle additive. An optimal ratio of the epoxy-polyester binder and additive was 100:0.06-0.08 pts. wt. It was ascertained that the introduction of pyrogenic silicon dioxide leads to a decrease in values of investigated properties of composite materials. In particular, the fracture surface of epoxy-polyester materials was analyzed with the method of optical microscopy. It was stated that composite materials filled with pyrogenic silicon dioxide were characterized by high residual stresses. The presence of low amount of fracture lines on the surface of composite materials filled with nanodispersed additive was revealed. Therefore, composite materials with nanofiller are characterized by higher resistance for fracture and impact loads.
\end{abstract}

Keywords: Composite material, Physical and mechanical properties, Transport.

DOI: 10.21272/jnep.13(1).01003

PACS number: $82.35 .-\mathrm{x}$

\section{INTRODUCTION}

The fast development of modern industry, in particular, the transport industry, leads to a continuous increase in requirements for the properties of used materials [1-6]. The increase in requirements is conditioned by the reliability of parts and mechanisms by reducing failure probability, their durability and reducing the inter-repair operational period [7-10]. Nowadays, polymers are the most widely used structural materials that are replacing metals and alloys [11-13]. The use of polymeric materials instead of metals increases the cost-effectiveness, reduces manufacturing costs and the weight of parts, with saving the physical-mechanical and thermophysical properties [14-18]. In this direction it was relevant to use polymeric materials based on epoxy and polyester components. Polymers based on epoxy and polyester resins are characterized by simplicity of formation and high physical-mechanical and thermophysical properties [19,20]. At the same time, the increase in requirements for the performance characteristics of polymers necessitates the search for new polymeric materials with improved properties. Therefore, the development of polymers with predetermined properties, which in complex will differ by improved physical and mechanical characteristics, is important from scientific and practical points of view.

As it is known $[8,14]$, polymeric materials are widely used as structural materials and coatings. Thermosetting materials are materials that are characterized by improved physical and mechanical properties. The authors [5] proved that the use of composites based on epoxy components is relevant. The study results of composite materials $(\mathrm{CMs})$ on the basis of polyester resins, which are also used in the shipbuilding and transport industry, are shown in the works $[13,17,19]$. The study results of $\mathrm{CMs}$, which combine two different in nature resins (epoxy and polyester) in one binder were presented in our previous works $[17,19]$. In particular, it should be noted that one of the ways to improve polymer properties is to introduce fillers of different nature and dispersion [14-16]. In the above stated works, the effect of fillers on the properties of $\mathrm{CMs}$ was investigated. It was proved that the use of nanofillers in small amount is expedient. At the same time, the introduction of nanofillers and high-dispersed microparticles allows to improve the properties of epoxy-polyester $\mathrm{CM}$ s and create materials with high reliability and durability for protecting parts of transport vehicles.

The objective of this work is to develop epoxypolyester CMs with improved physical and mechanical properties for increasing the reliability of transport vehicles.

\section{MATERIALS AND METHODS}

For the formation of CMs with increased physical and mechanical properties, the following components were used in the work:

1. The main component of the binder is a low mo-

\footnotetext{
*buketov.andrey@gmail.com
} 
lecular weight epoxy diane oligomer ED-20 ( $q=100$ pts. wt.) (ISO 18280:2010). It should be noted that the molecules of epoxy oligomers contain glycidyl and epoxy groups, which are capable, interacting with curing agent, of forming a cross-linked structure in the materials in a form of a grid [16].

2. Orthophthalic unsaturated pre-accelerated polyester resin Norsodyne O $12335 \mathrm{AL}$ ( $q=20$ pts. wt. per 100 pts. wt. of epoxy oligomer ED-20).

3 . The cold curing agent polyethylene polyamine (PEPA) $-q=10$ pts. wt. (the content is indicated per 100 weight parts of epoxy resin).

4. The initiator for polyester resins Butanox-M50 $q=1.5$ pts. wt. (the content is indicated per 100 weight parts of polyester resin Norsodyne O 12335), which is methyl ethyl ketone peroxide.

5 . An oxidized nanoparticle additive (ONA) with a particle size of $5-8 \mathrm{~nm}$. The chemical composition of the ONA: $57.23 \%$ of calcium carbonate $\left(\mathrm{CaCO}_{3}\right), 33.18 \%$ of carbon (graphite, carbon black), $9.59 \%$ of potassium chloride $(\mathrm{KCl})$.

6. Pyrogenic silicon dioxide (PSD) - highly dispersed, highly active, amorphous, pyrogenic silicon dioxide $\left(\mathrm{SiO}_{2}\right)$, which was obtained by high-purity flammable hydrolysis of silicon-chromium $\left(\mathrm{SiCl}_{4}\right)$ with a particle size of 7-12 nm.

The physical and mechanical properties of the CMs were investigated in order to identify the optimal concentration of fillers in the epoxy-polyester binder. The content of fillers was changing within the range of 0.02 1.0 pts. wt. per 100 pts. wt. of ED-20 epoxy resin.

The following physical and mechanical properties were investigated in this work: the elasticity modulus, flexural stresses at bending and the impact strength.

The flexural stresses at bending and the elasticity modulus at bending were determined according to ASTM D 790-03. The specimen parameters were as follows: length $l=120 \pm 2 \mathrm{~mm}$, width $b=15 \pm 0.5 \mathrm{~mm}$, height $h=10 \pm 0.5 \mathrm{~mm}$.

The impact strength was measured by pendulum copra according to Charpy method (ASTM D6110-18). We determined the working angle of deviation of the pendulum after the destruction of the specimen at a predefined initial angle of elevation of the working body of the testing machine. The investigation was conducted under temperature $T=298 \pm 2 \mathrm{~K}$ and relative humidity $d=50 \pm 5 \%$. We used specimens with the following sizes: $l \times b \times h=65 \times 12 \times 12 \pm 0.5 \mathrm{~mm}$.

The structure of fracture of the CMs was investigated additionally on a XJL-17AT metallographic microscope, which was equipped with a Levenhuk C310 NG (3.2 MegaPixels) camera. The specimens were examined with a magnification of $\times 400$ times. For the processing of digital images, Levenhuk ToupView software was used.

The epoxy-polyester composites with a nanofiller were formed according to the following technology with the implementation of temperature-time modes: preliminary dosage of ED-20 epoxy resin and Norsodyne O $12335 \mathrm{AL}$ polyester resin, heating of the binder and nanodispersed filler to a temperature of $T=353 \pm 2 \mathrm{~K}$, and keeping the components at a given temperature during time $t=0.3 \pm 0.02 \mathrm{~h}$; dosage of the filler and subsequent introduction into the binder; hydrodynamic combination of the oligomer ED-20 and the nanofiller over time $t=1 \pm 0.1 \mathrm{~min}$; ultrasonic treatment of the composition during time $t=1.5 \pm 0.1 \mathrm{~min}$; cooling of the composition to room temperature over time $t=1.0 \pm 0.08 \mathrm{~h}$; introduction of PEPA and Butanox-M50 curing agents and mixing of the composition over time $t=5 \pm 0.1 \mathrm{~min}$.

Further, the CMs were solidified according to the experimentally established regimen: formation of the specimens and their holding over time $t=12.0 \pm 0.1 \mathrm{~h}$ at a temperature of $T=293 \pm 2 \mathrm{~K}$, heating at a speed of $v=3 \mathrm{~K} / \mathrm{min}$ to a temperature of $T=393 \pm 2 \mathrm{~K}$, keeping the specimens at a given temperature during time $t=2.0 \pm 0.05 \mathrm{~h}$, and slow cooling to a temperature of $t=293 \pm 2 \mathrm{~K}$. In order to stabilize the structural processes in matrix, the specimens were kept in air during time $t=24 \mathrm{~h}$ at a temperature of $T=293 \pm 2 \mathrm{~K}$ followed by the conduction of the experimental tests.

\section{RESULTS AND DISCUSSION}

It was previously found that for the formation of a polymer matrix with improved physical and mechanical properties, the following components should be used in the complex: epoxy diane oligomer ED-20 $(q=100$ pts. wt. $)+$ Norsodyne O 12335 AL polyester resin ( $q=20$ pts. wt. per 100 pts. wt. of epoxy oligomer ED-20) + PEPA curing agent ( $q=10$ pts. wt. per 100 pts. wt. of ED-20) + curing agent for polyester resins - Butanox-M50 ( $q=1.5$ pts. wt. per 100 pts. wt. of polyester resin).

As it is known [5, 12], a significant effect in improving the CMs properties is observed when introducing nanofillers in homeopathic doses. At the initial stage, the effect of the content of the ONA nanofiller on the elasticity modulus during bending of epoxy-polyester CMs was investigated.

It was found that upon the introduction of the ONA nanofiller into the epoxy-polyester matrix (Fig. 1a, curve 1), the parameters of the elasticity modulus increased. In particular, it was noticeable that even at an insignificant content of particles ( $q=0.02 \mathrm{pts}$. wt.), the parameters of the elasticity modulus increase from $E=3.6 \mathrm{GPa}$ (for the matrix) to $E=3.8 \mathrm{GPa}$. Further, the parameters are unchanged with increasing content of the ONA particles to $q=0.1 \mathrm{pts}$. wt. Obviously, the ONA nanoparticles when interacting with the matrix can increase the degree of CM crosslinking, which affects the cohesive characteristics of the materials. Further, with an increase in the filler amount up to $q=0.25-0.50$ pts. wt., the parameters of the elasticity modulus gradually increase and the maximum value $E=4.2 \mathrm{GPa}$ is observed at the nanoparticles content of 0.05 pts. wt. It should be noted that ultrasonic dispersion was used for complete mixing of nanofiller particles throughout the composition volume. Obviously, in the complex, the ultrasonic cavitation of the binder and the presence of ONA particles in the epoxypolyester oligomer lead to a decrease in free radicals and activation of the physicochemical interaction of active centers on the surface of the nanofiller with groups and segments of the polymer. An increase in the nanofiller content within $q=0.5 \mathrm{pts}$. wt. leads to a decrease in the elas- 
ticity modulus parameters up to $E=3.5 \mathrm{GPa}$. Obviously, an increase in the content of these particles in the CM was not appropriate.

Analysis of the parameters of the elasticity modulus for bending obtained during the testing of epoxy-composite materials filled with PSD nanoparticles (Fig. 1a, curve 2) allows to state that the introduction of this filler does not affect the improvement of the CMs properties. When introducing PSD particles at a content of 0.02-0.06 pts. wt., an increase in the parameters of the CMs elasticity modulus within the experimental error $(\Delta E=0.1 \mathrm{GPa})$ was observed. Further, when increasing the number of nanoparticles, a decrease in the elasticity modulus from $E=3.7 \mathrm{GPa}$ to $E=3.5 \mathrm{GPa}$ was observed. Analyzing the dynamics of curve 1 and curve 2 in Fig. 1b, it can be stated that in the range of nanoparticles content of $\Delta q=0.02$ $0.06 \mathrm{pts}$. wt. the curves correlate. It is obvious that the increase in the parameters of the elasticity modulus in this range occurs due to the influence of ultrasonic dispersion, which confirms the previous assumptions. Then, an increase in the content of PSD particles over 0.6 pts. wt. leads to a deterioration of the elasticity modulus. Taking into account the chemical composition of the filler $\left(\mathrm{SiO}_{2}\right)$, it can be assumed that it is not active before interaction with $-\mathrm{OH},-\mathrm{CH}$ groups of the binder and supersaturation of this additive leads to a decrease in the elasticity parameters of the developed CMs [18, 21].

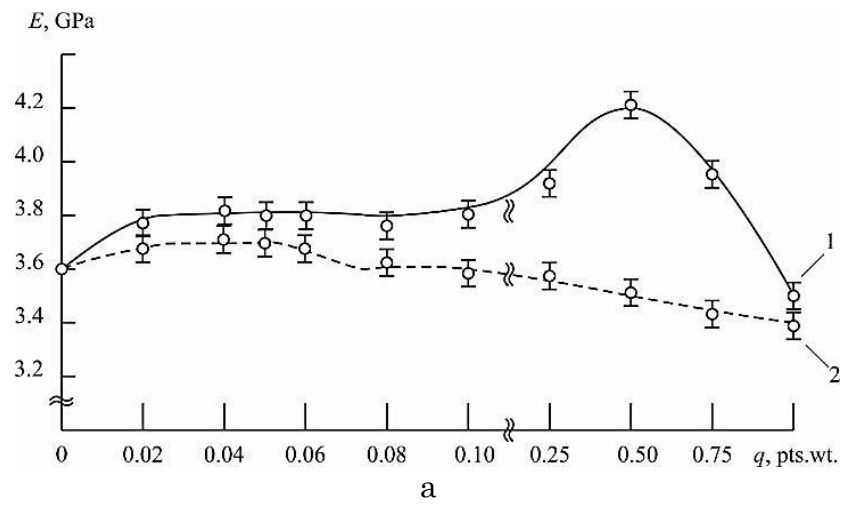

At the next stage, the influence of nanofiller particles on the flexural stresses of epoxy-polyester CMs was investigated. It was found (Fig. 1b) that the introduction of ONA nanoparticles at a content of $q=0.02$ pts. wt. leads to an increase in the value of flexural stresses from $\sigma_{b}=50.4 \mathrm{MPa}$ (for the matrix) to $\sigma_{b}=76.2 \mathrm{MPa}$. An increase in the ONA additives in the $\mathrm{CM}$ leads to a decrease in the parameters of flexural stresses at bending. In particular, at a content of particles of $q=0.04-1.00 \mathrm{pts}$. wt. the values are $\sigma_{b}=63.7$ 66.2 $\mathrm{MPa}$. When the additive content is greater than $q=1.0$ pts. wt., the parameters of flexural stresses at bending sharply decrease to the value $\sigma_{b}=26.3 \mathrm{MPa}$.

It should be noted that the results of the study of flexural stresses at bending with PSD particles (Fig. 2, curve 2) correlate with the parameters of the elasticity modulus of similar materials (Fig. 1b, curve 2). Introduction of PSD particles leads to deterioration of CM properties and the parameters of flexural stresses are gradually reduced from $\sigma_{b}=50.4 \mathrm{MPa}$ to $\sigma_{b}=18.5 \mathrm{MPa}$ with increasing content of nanoparticles. Such materials are characterized by increased fragility. It should be noted that the particles of pyrogenic silicon dioxide $\left(\mathrm{SiO}_{2}\right)$ have the property of hygroscopicity [21]. Therefore, it is obvious that this effect leads to a decrease in the parameters of materials flexural stresses.

Fig. 1 - Dependences of the elasticity modulus at bending (a) and flexural stresses at bending (b) of the epoxy-polyester CM on the content of nanoparticles, $q$, pts. wt.: 1 - ONA; 2 - PSD

Subsequently, the impact strength of nanofilled composites was investigated. It was found (Fig. 2, curve 2) that the PSD particles, as well as in the study of the previous properties of $\mathrm{CMs}$, lead to a decrease in the parameters of impact strength of composites. This confirms previous assumptions that the hygroscopicity of this filler causes increased fragility of materials. The parameters of the impact strength with the increasing content of nanoparticles are gradually reduced from $W=8.3 \mathrm{~kJ} / \mathrm{m}^{2}$ (for the matrix) to $W=1.7 \mathrm{~kJ} / \mathrm{m}^{2}$ (for the $\mathrm{CM}$ filled with PSD particles at a content of $q=1.0$ pts. wt.).

It was found (Fig. 2) that when introducing ONA filler at a content of $q=0.02 \mathrm{pts}$. wt., the value of the impact strength decreases from $W=8.3 \mathrm{~kJ} / \mathrm{m}^{2}$ (for the matrix) to $W=5.8 \mathrm{~kJ} / \mathrm{m}^{2}$. Subsequently, the staged introduction of particles of the nanofiller provides a monotonous increase in the impact strength parameters, and the maximum values $\left(W=8.3 \mathrm{~kJ} / \mathrm{m}^{2}\right)$ have $\mathrm{CM}$ at the additive content of $q=0.06-0.08$ pts. wt. Further addition of ONA particles leads to a decrease in the impact strength of the material to $W=4.2 \mathrm{~kJ} / \mathrm{m}^{2}$ (at the content $q=1.0 \mathrm{pts}$. wt.).

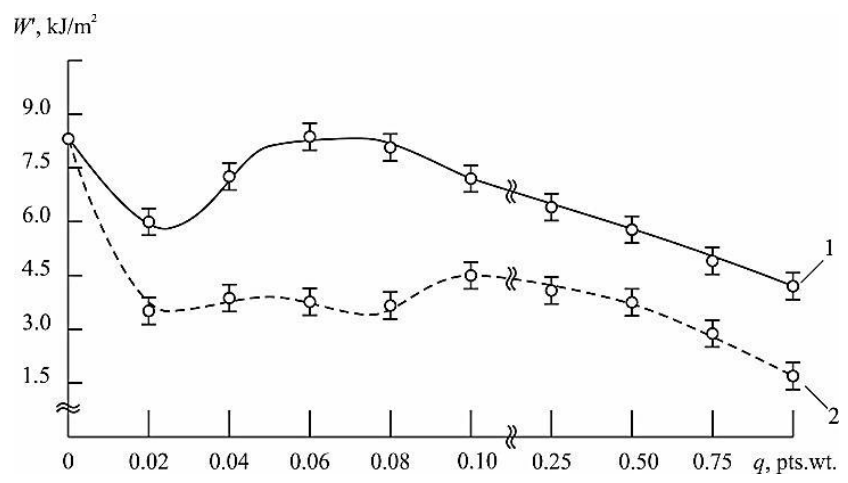

Fig. 2 - Dependence of the impact strength $(W)$ of epoxy-polyester $\mathrm{CM}$ on the content of nanoparticles, $q$, pts. wt.: 1 - ONA; $2-\mathrm{PSD}$ 
Consequently, analyzing the results obtained on the parameters of the properties of the elasticity modulus and flexural stresses at bending and the impact strength, it can be stated that the CMs filled with ONA particles at a content of $q=0.06-0.08$ pts. wt. are characterized by improved properties. At the same time, the value of the elasticity modulus at bending of the developed materials is $E=3.8 \mathrm{GPa}$, the flexural stresses at bending are $\sigma_{b}=63.7 \mathrm{MPa}$, and the impact strength is $W=8.3 \mathrm{~kJ} / \mathrm{m}^{2}$.

At the final stage, the fracture surface of epoxy CMs was analyzed (Fig. 3). Studying fracture patterns of the matrix fracture (Fig. 3a) it was noticeable that the cracks are distributed chaotically throughout the surface, the relief and cracking lines of the material are clearly visible. The resulting fracture distribution at the point of fracture indicates a high-voltage state of the CM. This was confirmed by the above-obtained parameters of the impact strength of the matrix.

During the study of the fracture surface of the CM filled with ONA nanoparticles (Fig. 3b-g) it was observed that the nature of the crack distribution is uniform, the lines are straight and their depressions do not differ significantly. In particular, it should be noted that on the fracture patterns of the CM at ONA content of 0.075-0.10 pts. wt. (Fig. 3d, e) the surface differs by the minimum number of shearing lines, in comparison with other specimens, without branching and with insignificant furrows. This indicates that this content of the nanofiller leads to optimal cross-linking of CM in the polymer-filler system. The formed bonds between the matrix and the filler allow leveling the stress state of the heterogeneous system and as a result, a stable polymer for cracking and impact shocks was obtained.
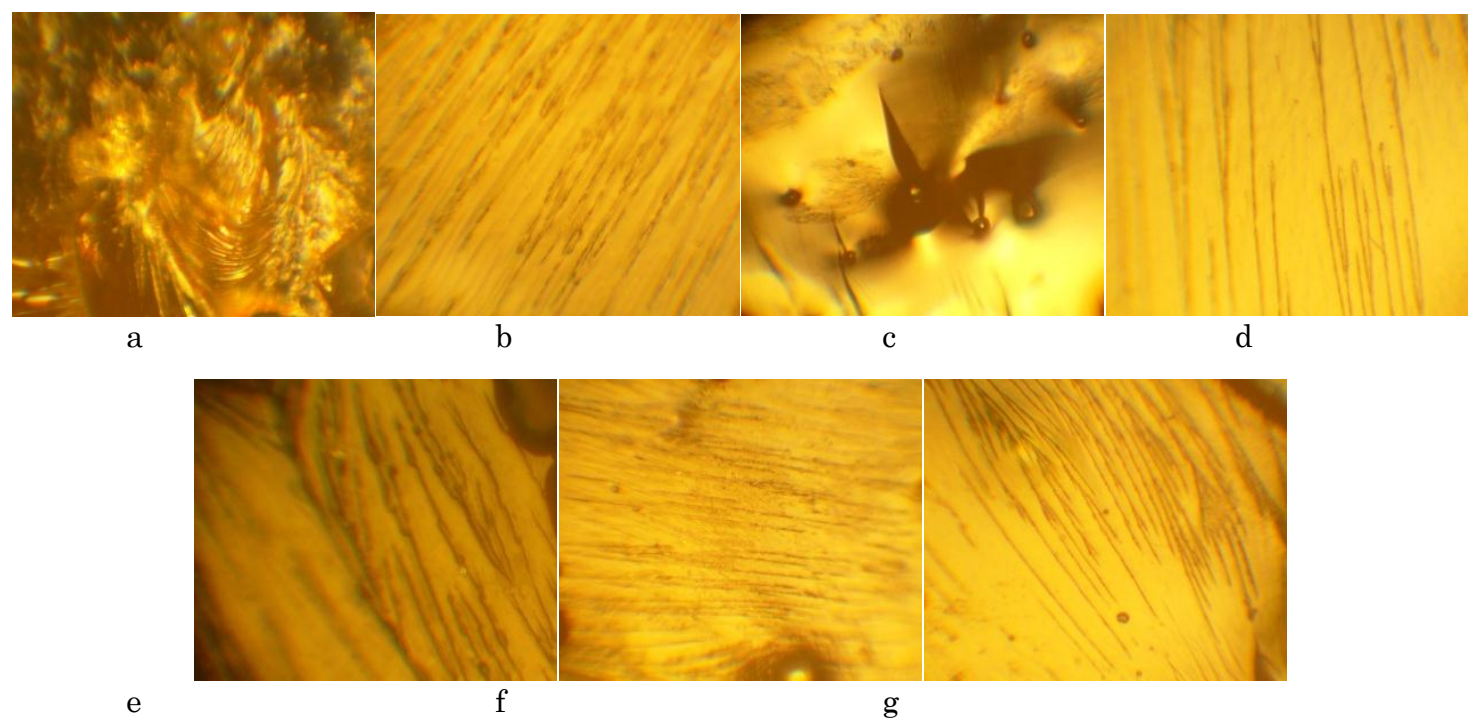

Fig. 3 - Fracture patterns of CM filled with ONA particles at different content: a) matrix; b) $q=0.025$ pts. wt.; c) $q=0.05$ pts. wt.; d) $q=0.075$ pts. wt.; e) $q=0.1$ pts. wt.; f) $q=0.5$ pts. wt.; g) $q=1.0$ pts. wt.

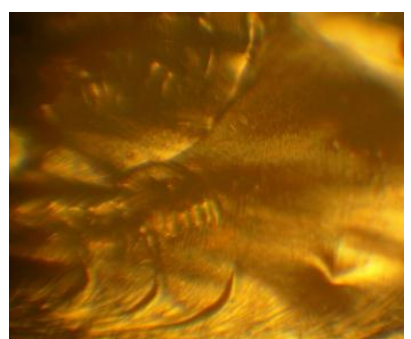

a

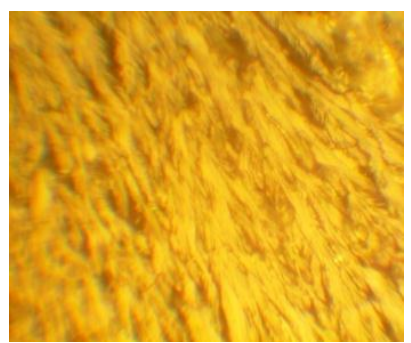

d

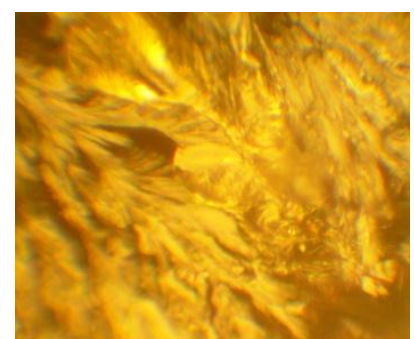

$\mathrm{b}$

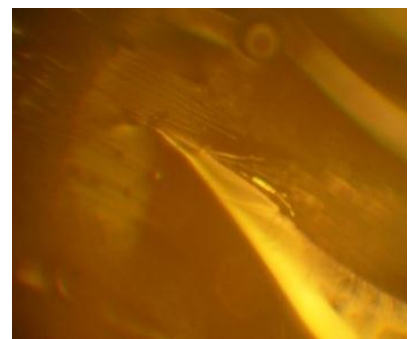

e

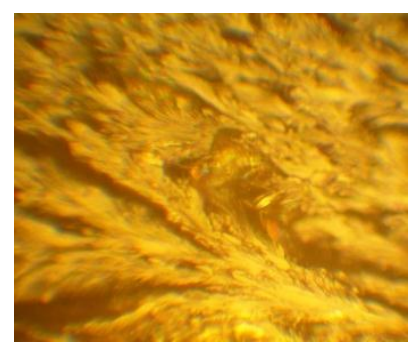

$\mathrm{c}$

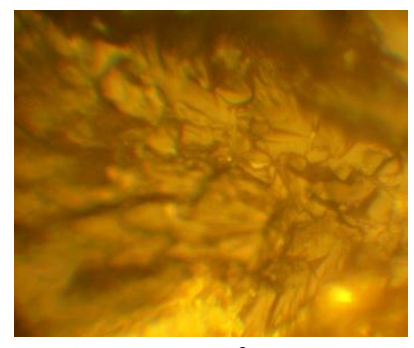

f

Fig. 4 - Fracture pattern of CM filled with PSD particles at different content: a) $q=0.025$ pts .wt.; b) $q=0.05$ pts. wt.; c) $q=0.075$ pts. wt.; d) $q=0.1$ pts. wt.; e) $q=0.5$ pts. wt.; f) $q=1.0$ pts. wt. 
Analysis of the pattern of fracture surface of $\mathrm{CMs}$ filled with PSD particles (Fig. 4) allows to state that the overall shearing lines are pronounced, and considerable depressions of cracks propagation are chaotic. Such a nature of the material surface after fracture indicates a high residual stress in such CMs. It was obvious that the presence of PSD particles leads to the formation of stress concentrators, which, as a consequence, influence the performance of physical and mechanical properties of the CMs.

Consequently, the results of the analysis of the fracture surface of CMs filled with ONA and PSD nanoparticles completely correlate with the parameters of the physical and mechanical properties of the materials, indicating the reliability of the data.

\section{CONCLUSIONS}

Based on the results of experimental studies, one can state the following.

It was established that the introduction of nanoparticles of the ONA filler influences the physical and mechanical properties of epoxy-polyester CMs. It was proved that composites filled with ONA particles at a content of $q=0.06-0.08$ pts. wt. are characterized by improved properties. At the same time, the value of the elasticity modulus at bending of the developed materials is $E=3.8 \mathrm{GPa}$, the flexural stresses at bending are $\sigma_{b}=63.7 \mathrm{MPa}$ and the impact strength is $W=8.3 \mathrm{~kJ} / \mathrm{m}^{2}$.

It was analyzed that the introduction of PSD particles leads to a decrease in the parameters of physical and mechanical properties of polymer composites. At

\section{REFERENCES}

1. A. Panda, K. Dyadyura, M. Harničárová, J. Valíček, I. Pandová, Z. Palková, MM Sci. J. 3500 (2019).

2. A. Panda, K. Dyadyura, P.P. Savchuk, V. Kashytskyi, V. Malets, J. Valíček, M. Harničárová, I. Pandová, L. Androvič, M. Kušnerová, MM Sci. J. 3509 (2019).

3. I.M. Pohrelyuk, M.V. Kindrachuk, S.M. Lavrys', Mater. Sci. 52, 56 (2016).

4. A.S. Mostovoy, A.S. Nurtazina, I.N. Burmistrov, Y.A. Kadykova, Russ. J. Appl. Chem. 91, 1758 (2018).

5. P.P. Savchuk, V.P. Kashytsky, O.L. Sadova, O.M. Lyushuk, Powder Metall. Met. Ceram. 56, 448 (2017).

6. V. Zaloga, K. Dyadyura, I. Rybalka, I. Pandova, Manag. Syst. Prod. Eng. 27, 221 (2019).

7. V.Y. Panarin, M.Y. Svavil'nyy, A.I. Khominych, M.V Kindrachuk, A.O. Kornienko, J. Nano- Electron. Phys. 9 No 6, 06023 (2017).

8. I.N. Burmistrov, A.S. Mostovoi, N.V Shatrova, L.G. Panova, D.V Kuznetsov, A.V Gorokhovskii, I.A. Il'inykh, Russ. J. Appl. Chem. 86, 765 (2013).

9. V.G. Efremenko, K. Shimizu, A.P. Cheiliakh, T.V Pastukhova, Y.G. Chabak, K. Kusumoto, Int. J. Miner. Metall. Mater. 23, 645 (2016).

10. L.F. Sukhodub, K. Dyadyura, J. Nano- Electron. Phys. 10 No 6, 06003 (2018).

11. V. Efremenko, K. Shimizu, T. Pastukhova, Y. Chabak, M. Brykov, K. Kusumoto, A. Efremenko, Int. J. Mater. Res. 109, 147 (2018). the same time, the parameters of the elasticity modulus at bending are gradually reduced from $E=3.6 \mathrm{GPa}$ to $E=3.4 \mathrm{GPa}$, the flexural stresses at bending from $\sigma_{b}=50.4 \mathrm{MPa}$ to $\sigma_{b}=18.5 \mathrm{MPa}$, the impact strength from $W=8.3 \mathrm{~kJ} / \mathrm{m}^{2}$ to $W=1.7 \mathrm{~kJ} / \mathrm{m}^{2}$.

The fracture surface of composites was analyzed by the method of optical microscopy, and the results of physical and mechanical properties are confirmed. It was established that the cracks propagation on the fracture pattern of CMs filled with PSD particles was characterized as composites with high residual stresses. In the study of the fracture of composites filled with ONA particles at a content of $q=0.075-0.10$ pts. wt., it can be stated that the surface differs by the minimum number of shearing lines in comparison with other specimens, without branching and with insignificant furrows. This indicates that this content of the nanofiller leads to optimal cross-linking of $\mathrm{CMs}$ in the system polymer/filler. The formed bonds between the matrix and the filler allow to level the stress state of the heterogeneous system and, as a result, a polymer resistant to cracking and impact shocking was obtained.

\section{ACKNOWLEDGEMENTS}

The publication contains the results of research conducted within the framework of the project 2020.02/393 "Development of nanopolymer composites for the restoration of the main mechanisms and bodies of water and land transport" funded by the National Research Fund of Ukraine from the state budget.

12. M.V Kindrachuk, D.A. Vol'chenko, N.A. Vol'chenko, N.M. Stebeletskaya, A.V. Voznyi, Mater. Sci. 53, 282 (2017).

13. B. Duleba, F. Greškovič, E. Spišák, L. Dulebová, $A d v$. Mater. Res. 132 (Trans Tech Publ: 2014).

14. V.P. Privalko, R.V Dinzhos, E.G. Privalko, Thermochim. Acta 432, 76 (2005)

15. K.A. Dyadyura, K.V Berladir, P.V Rudenko, O.A. Budnik, V.A. Sviderskij, 2016 Int. Conf. Nanomater.: Appl. Prop. (NAP-2016) 02NSA04 (2016).

16. A.V. Buketov, N.A. Dolgov, A.A. Sapronov, V.D. Nigalatii, N.V. Babich, Strength Mater. 49, 464 (2017).

17. A.V. Buketov, P.D. Stukhlyak, I.G. Dobrotvor, N.M. Mytnyk, N.A. Dolgov, Strength Mater. 41, 431 (2009).

18. A. Buketov, M. Brailo, S. Yakushchenko, A. Sapronova, Adv. Mater. Sci. Eng. 2018, 1 (2018).

19. O.O. Sapronov, A.V. Buketov, P.O. Maruschak, S.V. Panin, M.V. Brailo, S.V. Yakushchenko, A.V. Sapronova, O.V. Leshchenko, A. Menou, Funct. Mater. 26, 114 (2019).

20. A. Buketov, M. Brailo, S. Yakushchenko, O. Sapronov, V. Vynar, O. Bezbakh, R. Negrutsa, Period. Polytech. Mech. Eng. 63, 171 (2019).

21. A.V. Akimov, A.V. Buketov, A.A. Sapronov, M.V. Brailo, S.V. Yakushchenko, S. A. Smetankin, Compos. Mech. Comput. Appl. An Int. J. 10, 117 (2019).

22. M. Zienkiewicz-Strzałka, M. Błachnio, A. DeryłoMarczewska, R.B. Kozakevych, Y.M. Bolbukh, V.A. Tertykh, Adsorpt. Sci. Technol. 35, 714 (2017). 